\title{
Antichlamydial Activity of Tear Fluid
}

\author{
A. N. ELBAGIR, ${ }^{1}$ K. STENBERG,${ }^{2}$ G. FRÖMAN,${ }^{1}$ P-A. MÅRDH, ${ }^{1}$ \\ Sweden
}

\begin{abstract}
Summary
Tear fluid collected from healthy children and adults, was tested for its capacity to inhibit Chlamydia trachomatis, serotype I, to form inclusions in McCoy cell cultures. Pooled tear fluid added to such cultures reduced the chlamydial inclusion count even at concentrations of $1 \%$. The inhibitory activity was concentration-dependent. The chlamydial inhibitory factor has a molecular weight of $<10000$ dalton and the principle is heat-stable. The antichlamydial factor seems to affect the attachment of the elementary body (EB) to the host cell surface, while no effect on the intracellular development and reproduction of the chlamydiae could be demonstrated. The activity could not be explained by the presence of antichlamydial antibodies.
\end{abstract}

The external eye is protected in cases of infection by a number of local defence mechanisms. As a response to external eye infections $\operatorname{IgA}{ }^{1}$ can occur in higher concentrations in the lacriminal fluid. The lysozyme concentration in tear fluid may vary during infection and, at least in vitro, it can lyse certain Gram-positive bacteria. ${ }^{2}$ A heat-labile component of tears caused growth-inhibition of staphylococci. ${ }^{3} \mathrm{~A}$ dialysable, heat-labile non-lysozyme antibacterial factor (NLAF) with a molecular weight of 1500-5000, has also been demonstrated in tear fluid. ${ }^{4}$ Subsequently, it was suggested that NLAF was $\beta$-lysin, ${ }^{5}$ which is a heat-stable bacterial factor found in platelets, human serum and certain other body fluids. ${ }^{6}$ Other antimicrobial compounds demonstrated in tear fluid include lactoferrin ${ }^{7}$ and a specific tear prealbumin. $^{8}$

Body fluids, such as amniotic fluid, ${ }^{9,10}$ and seminal plasma ${ }^{11}$ and some of its constituents, e.g. spermine and zinc ions, might interfere with the capacity of Chlamydia trachomatis to form inclusions in McCoy cell cultures. So far, to the best of our knowledge, there have been no reports on an antichlamydial activity (nonIg-related) of tear fluid.

The purpose of our present study was to test tear fluid of healthy individuals for possible antichlamydial activity.

\section{Materials and Methods}

\section{Collection of tear fluid}

Tear fluid was obtained from the eyes of healthy subjects within the age range of one to 40 years. Tears were collected in one of the following ways:

(i) Naturally stimulated tear production induced by the natural process of yawning. Between 0.5 and $1.0 \mathrm{ml}$ tear fluid could be obtained from a subject at any one time.

(ii) Indirectly stimulated tear-production was provoked by inserting a swab into the nasopharynx. By this method only 10 to $100 \mu \mathrm{l}$ of tear fluid could be obtained at a time.

In both methods, tears were sucked into microcapillary pipettes without touching the conjunctivae. The tears were pooled in sterile glass vials and stored at -70 degree $C$, until use.

From: Institute of Clinical Bacteriology, ${ }^{1}$ University of Uppsala, Uppsala, and Department of Ophthalmology, ${ }^{2}$ University of Göteborg, Sweden.

Correspondence to: Dr. Adil Norein Elbagir, Institute of Clinical Bacteriology, University of Uppsala, Box 552, S-751 22 Uppsala, Sweden. 
Washing fluid from pipettes and vials were tested for possible antichlamydial properties. Culture medium that had been preincubated overnight at 37 degree $C$ was added to the culture system and compared to controls.

Local or systemic antibiotic therapy had not been given to any of the subjects studied for at least two weeks prior to sampling.

\section{Organism}

A strain of Chlamydia trachomatis, serotype I (obtained from Dr SP Wang, Seattle, WA), that had been passed several times in McCoy cell cultures before being tested, was used.

\section{Culture technique}

The effect of tear fluid on the inclusion formation capacity of the chlamydial test strain was studied using cycloheximide-treated McCoy cell cultures as previously described. ${ }^{12} \mathrm{Com}$ plete medium glucose antibiotic (CMGA), consisting of RPMI 1640 (Flow Lab. Ltd., Scotland) with $10 \%$ inactivated fetal calf serum, $30 \mathrm{mM}$ glucose, $50 \mathrm{mg} / \mathrm{ml}$ gentamycin and $2 \mathrm{mM}$ glutamine was used as culture medium as well as diluent for the tear fluid. The cells were seeded with $1 \mathrm{ml}$ of a concentration of $2 \times 10^{5} \mathrm{cell} / \mathrm{ml}$ and incubated for $24 \mathrm{~h}$ at 37 degree $\mathrm{C}$ in an atmosphere of $10 \% \mathrm{CO}_{2}$. CMGA with and without chlamydiae was added $(0.6 \mathrm{ml})$ to wells of 24-well culture plates, containing McCoy cells cultures. Pooled tear fluid was added to the chlamydial mixtures as follows:

(i) Different concentrations of tear fluid in CMGA all containing the same concentrations of chlamydiae were preincubated at 37 degree $\mathrm{C}$ for 30 minutes in an atmosphere of $10 \% \mathrm{CO}_{2}$, and maintained in the cell culture for the first two hours of incubation.

(ii) Tear fluid mixtures were added to the infected cell cultures for the last 48 hours.

(iii) Tear fluid at concentration of $5 \%$ in CMGA was incubated at 37 degree $\mathrm{C}$ for three hours or overnight in $10 \% \mathrm{CO}_{2}$ in the presence of chlamydiae before centrifugation of the mixture at $7200 \times \mathrm{g}$ for one hour. The deposit was then added to McCoy cell cultures. Experiments following the same test procedures, but without tear fluid were used as controls. All the tests were done in triplicate. The results are given as the mean of the three deter- minations. The McCoy cells were fixed in $96 \%$ ethanol, stainedwith labelled monoclonal antibodies to Chlamydia trachomatis (Syva) and examined under a fluorescence microscope. The number of chlamydial inclusions (CI) was determined and the morphology of the tissue culture cells noted.

To exclude nutrient deficiency of the culture medium (due to the dilution by tear fluid) as a cause of a demonstrated antichlamydial activity, experiments using double strength of CMGA were used in tests having $25 \%$ concentration of tear fluid.

Experiments were performed to test the effect of heating on the antichlamydial activity, using tear fluid exposed to 90 degree $\mathrm{C}$ for 15 minutes.

Ultrafiltration of tear fluid was made through a low-adsorption, hydrophilic YM membrane with molecular weight cut-off of 10000 (Amicon \& Grace Co., Sweden) combined with centrifugation at $1700 \times \mathrm{g}$ for one hour. The ultrafiltrate was tested for its effect on the CI formation, as described above.

A microimmunofluorescence test was used to detect antibodies to $C$. trachomatis in the tear fluid studied. Titres of 1:4 for IgA and 1:16 for IgG antibodies were used as cut-off levels. An antigen pool of $C$. trachomatis, consisting of serotypes of D through $\mathrm{K}$, was used. ${ }^{13} \mathrm{An}$ enzyme linked immunosorbant assay (ELISA) was also used for the detection of antichlamydial antibodies. Briefly, a 96-well microtiter plate was coated with $1 \mu \mathrm{g} /$ well of $C$. trachomatis serotype I. A $1 \%$ polyethylenglycol (MW 20000) solution in phosphate buffered saline (PBS) was used in order to block non-specific binding. Secondary antibodies conjugated with peroxidase (Dakopatts, Denmark) were added and after incubation at 37 degree $\mathrm{C}$ for 45 minutes the substrate (orthophenylenediamine, Abbotts) was added and absorbance measured at $492 \mathrm{~nm}$.

To study the effect of tear fluid on the adhesion of the chlamydiae to McCoy cells, coverslip cultures were made as indicated above, to which tears and chlamydiae were added. Following centrifugation and two hours incubation, the McCoy cells were washed twice with RPMI and fixed with $96 \%$ ethanol. The cells were then washed in phosphate buffer saline (PBS) and distilled in $\mathrm{H}_{2} \mathrm{O}$, stained with 
monoclonal antibodies (MikroTrak, SYVA) and examined for EBs under a fluorescence microscope.

\section{Results}

The inhibitory effect of tear fluid on CI formation is demonstrated in Figure 1. Tear fluid when present during the first two hours in the chlamydial culture system produced a concentration-dependent reduction in the inclusion count. A $50 \%$ inhibition of the CI count was obtained in the presence of less than $10 \%$ concentration of tear fluid.

As shown in Figure 1, the use of double strength CMGA in tests using a $25 \%$ concentration of tear fluid did not result in a significant change in its inhibitory activity.

No cytopathological effect was seen when tears were incorporated in the cell culture, at a concentration of $25 \%$ or less. At higher concentrations this was seen, as cells loosening from the wells and clumping together.

The inhibitory effect seems to involve only the initial stage of the chlamydial infection of the McCoy cells, since there was an effect only when the tear fluid was present during the first two hours of infection. Adding tears after the first two hours of infection gave no inhibitory effect (Fig. 2).

Treating the tear fluid by heat ( 90 degree $\mathrm{C}$ for 15 minutes) or ultrafiltration (cut-off $\mathrm{MW}$ 10000 daltons) did not alter the inhibitory activity (Fig. 3).

Overnight incubation of tears with chlamydiae resulted in no $\mathrm{CI}$ even in control cultures. With three hours' preincubation of tears and chlamydiae, a $65 \%$ reduction in the $\mathrm{CI}$ count compared to the controls was seen.

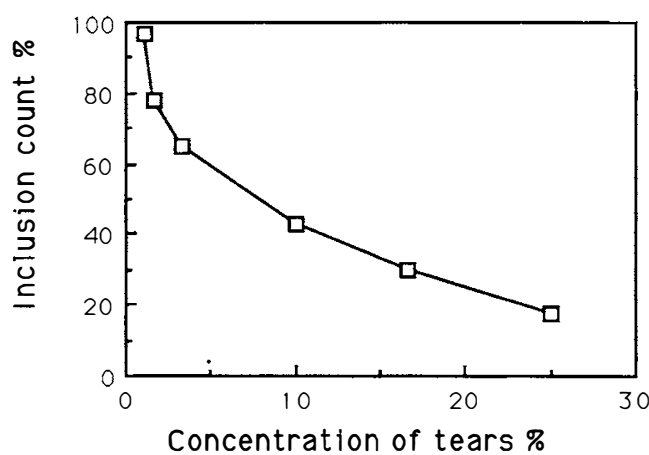

Fig. 1. Effect of tear fluid on inclusion count of Chlamydia trachomatis in McCoy cell cultures. Results expressed as percentage of controls.
By MIF tests or ELISA (Fig. 4), no IgA or IgG antichlamydial antibodies were detected in the tear fluid tested.

When culture medium was preincubated in the kind of test tubes used in our experiments, a $5 \%$ reduction in the $\mathrm{CI}$ count as compared to the controls was seen.

Tear fluid inhibited the attachment of chlamydial elementary bodies(EBs) to the McCoy cells. As shown in Figure 5, in the control cultures EBs are closely adherent to McCoy cells, whereas in the tear-containing cultures, the EBs are seen scattered in-between the cells.

\section{Discussion}

In the present study, we demonstrated the existence of a concentration-dependent inhibition of the capacity of $C$. trachomatis to form inclusions in McCoy cell cultures, in the presence of tear fluid collected from healthy individuals. Concentrations of tears as low as $1 \%$ caused a $20 \%$ reduction of the CI count as shown in Figure 1.

It has previously been reported that a significant increase in the serum proteins level is seen when Schirmer's filter papers were used for collection of tears, as compared to the use of micro-capillary-pipettes. ${ }^{14}$ The collection techniques we used excluded possible exudation of serum proteins, such as albumin, IgG and transferrin.

We also did tests to rule out the possibility that washing fluids used contained toxic components responsible for the demonstrated antichlamydial activity. Other studies have

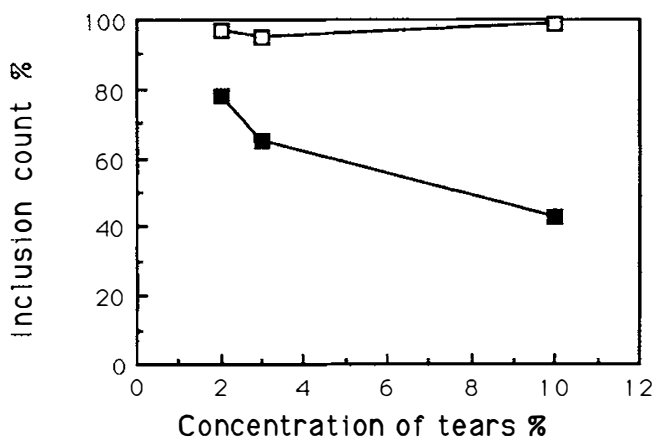

Fig. 2 Effect of tear fluid on inclusion count of Chlamydia trachomatis in McCoy cell cultures when tears are present for first two hours ( $\square$ ), and for last 48 hours of incubation ( $\square$ ). Results expressed as percentage of controls. 


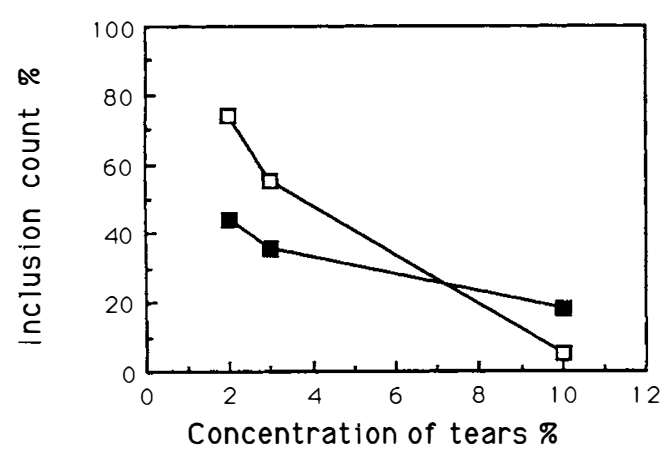

Fig. 3. Effect of tear fluid heated at 90 degree $C$ for 15 minutes (घ). And of ultrafiltration ( $\square$ ), on inclusion count of Chlamydia trachomatis. Results expressed as percentage of controls.

demonstrated that some washing fluids contain toxic substances to chlamydiae. ${ }^{15}$

The incubation of the chlamydial culture system for 48 hours or longer was associated with rupture of the chlamydial inclusions and dispersion of the EBs. This phenomenon made the interpretation of the results difficult, so for accuracy, such design of experiments should use shorter incubation periods.

Tear fluid had no cytopathologic effect (CPE) on the McCoy cells, except when concentrations of $25 \%$ or greater were used. This suggests that tears at lower concentration have an effect on the EB rather than on the cells.

The dilution effect on the culture medium by the addition of tears, as a cause of the demonstrated antichylamydial activity was ruled out by the use of double strength CMGA in tests with high concentrations of tear fluid.

Three hours' preincubation of chlamydiae with tears resulted in a reduction of the CI count, suggesting a toxic effect of tears on the chlamydiae. The exact mechanism of this effect has still to be established. However, an enzymatic activity in tears acting on the chlamydial membrane components could be a factor. Adding tears only for the first two hours of incubation caused the dose-related reduction of CI count (Fig. 2). This suggests that tears may interfere with the integrity of the EB as well as its attachment to host cells, as demonstrated in Figure 5.

Thirty-two thousand and 18000 dalton proteins act as adhesions of EBs to host cells. Also protease inhibitors, e.g. antitrypsin and antichymotrypsin, blocked chlamydial infectivity of the host cells. ${ }^{16}$ Other authors reported that such protease inhibitors are present in small quantities in tears. ${ }^{17}$ This might explain the demonstrated antiattachment effect of tears on the chlamydiae-host interaction. When tears were added after two hours incubation, and maintained through the rest of the growth cycle no effect on the CI count wasseen. This seems to indicate that tears may not have an inhibitory role on the intracellular reproduction of EBs.

Several substances with antibacterial activity, including $\operatorname{IgA}$, lysozyme, ${ }^{2} \beta$-lysin, ${ }^{5}$ lactoferrin and transferrin ${ }^{7}$ and a specific tear prealbumin (STP) have been identified in tear fluid.

The antichlamydial factor we report on differs in many aspects from the above mentioned compounds. Our demonstrated antichlamydial factor is heat-stable, has a molecular weight of $<10000$ dalton, and is not an antichlamydial antibody. The heat stability of our factor suggests its non-proteinous nature. Lysozyme at certain concentrations has been found to stimulate rather than inhibit inclusion formation of $C$. trachomatis. ${ }^{11}$ Lactoferrin is a compound with a molecular weight of 82000 dalton. $^{7}$

Purified $\beta$-lysin showed a complex protein mixture composed of more than 45 bands on sodium dodecyl sulfate gradient gels, ${ }^{18}$ and a lot of dispute about its biochemical nature has been reported. ${ }^{4,5}$ Our demonstrated factor does not seem to be of a proteinous nature. Transferrin is an iron-binding protein with a molecular weight of about 80000 dalton. ${ }^{8} \mathrm{STP}$,

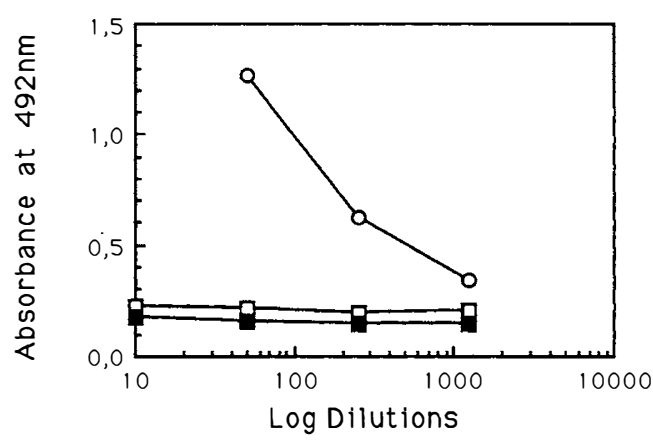

Fig. 4. $\operatorname{Ig} G(\square)$ and $\operatorname{Ig} A$ ( $\square)$ antibodies to Chlamydia trachomatis in tear fliud tested by an ELISA method. Results compared to serum containing pooled IgG and Ig $A$ antichlamydial antibodies (O). 


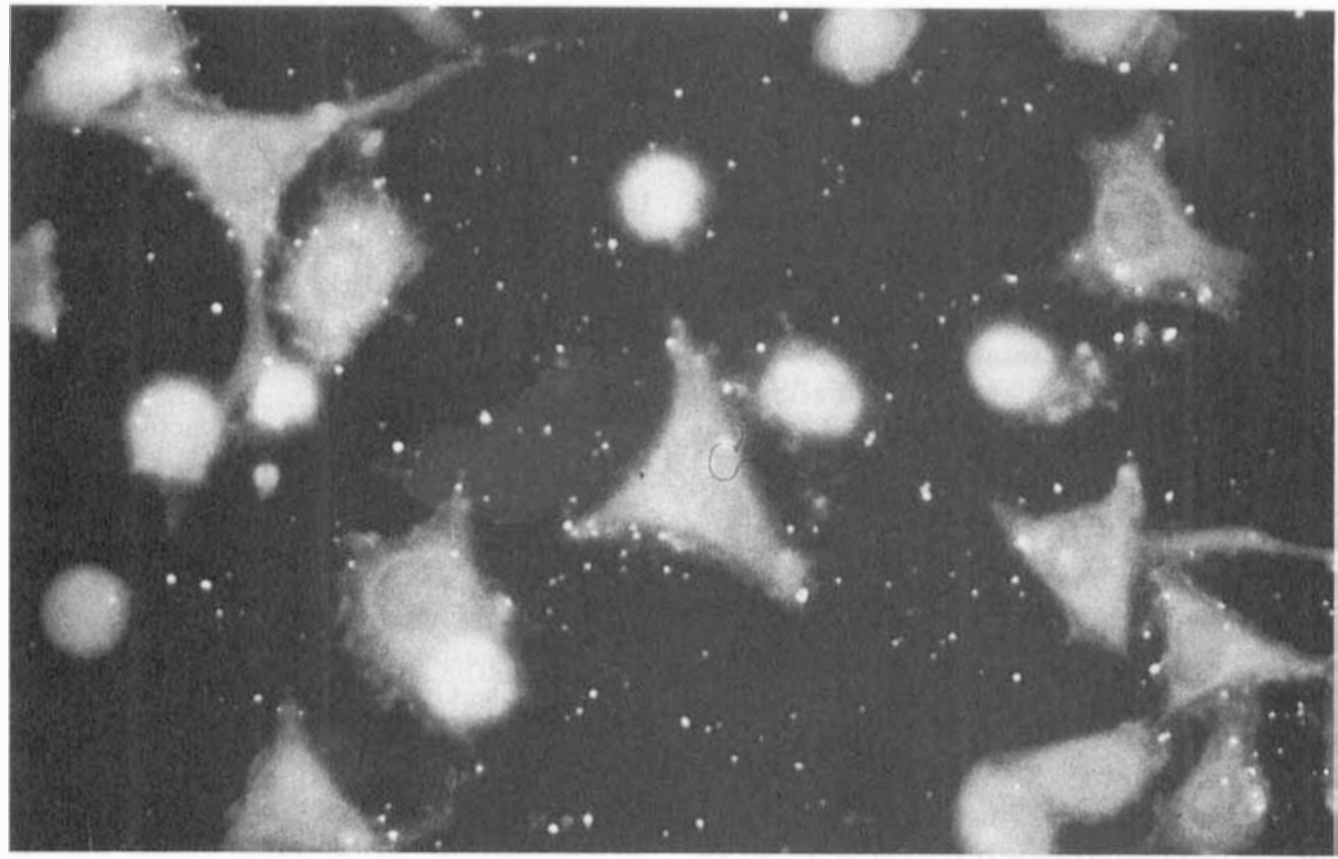

Fig 5a

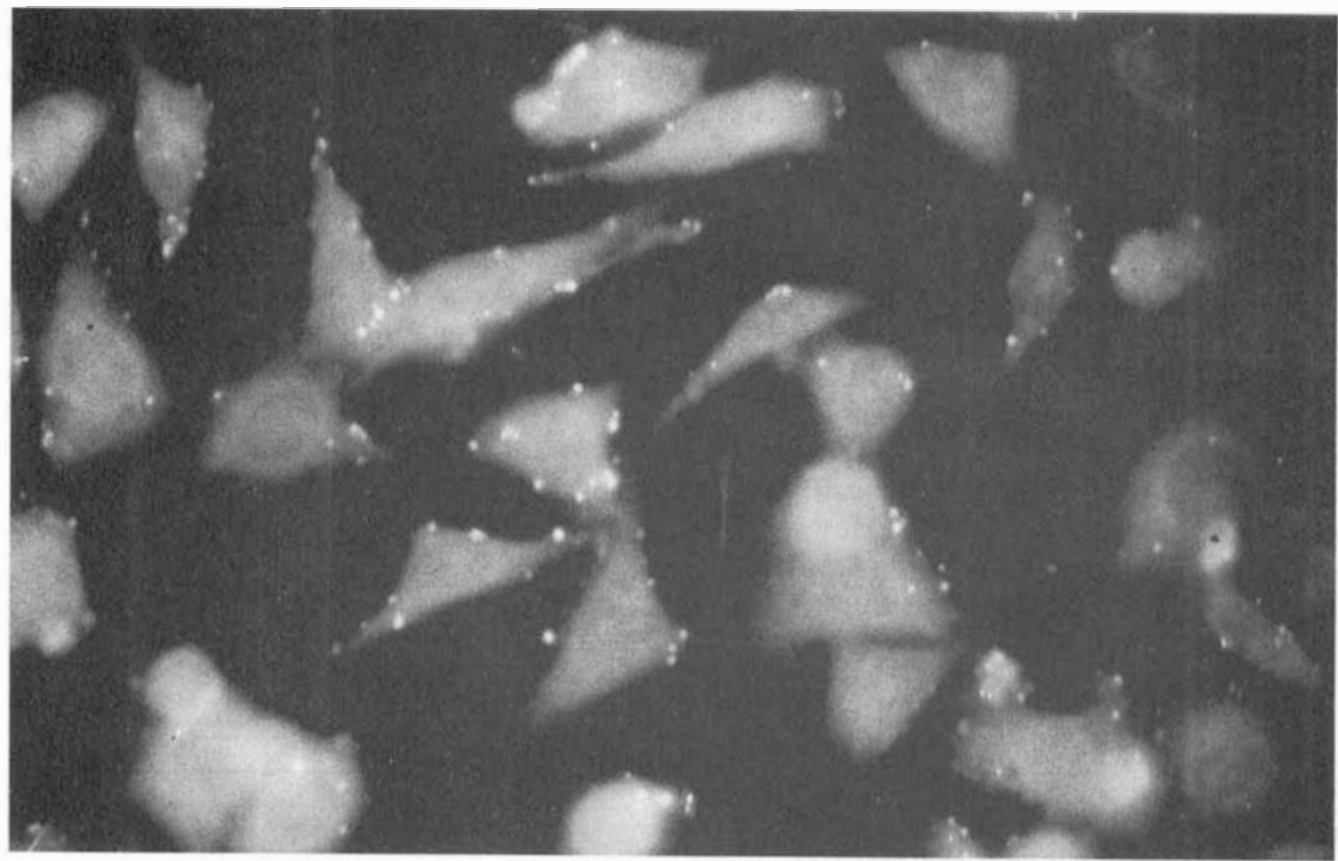

Fig. 5b

Fig. 5 Effect of tear fluid on Chlamydia trachomatis attachment to McCoy cells, in the presence of $10 \%$ concentration of tears (a) as compared to the control culture (b). 
on the other hand, showed an electrophoretic pattern with two bands, each corresponding to a molecular weight of $>10000$ dalton. ${ }^{8}$ These higher molecular weight substances are thus excluded as they may be the antichlamydial principle in tear fluid.

The antichlamydial principal in tear fluid seems to be similar to that demonstrated in amniotic fluid. ${ }^{9,10}$ We have also demonstrated that components of body fluids, such as $\mathrm{Zn}^{++}$ ions, caused a concentration-dependent reduction of the CI count in the McCoy cell cultures. ${ }^{11}$ Other workers have also shown that exposure of the chlamydial culture system to $\mathrm{Zn}^{++}$ions for 30 to 60 minutes, augmented infection by $C$. trachomatis, while more prolonged exposure ultimately killed the chlamydiae. ${ }^{19}$ Whether $\mathrm{Zn}^{++}$ions may play a role in the demonstrated antichlamydial activity, remains to be established.

The existence of an antichlamydial principal in tear fluid might explain why chlamydial conjunctivitis is a comparatively rare manifestation of $C$. trachomatis in cases with genital chlamydial infection. It has been estimated that only 1 in 200 to 300 genitally infected patients, will acquire conjunctivitis. The transmission to the eye is believed to be by autoinoculation.

The demonstrated antichlamydial activity of tears can be an important resistance mechanism against chlamydial eye infections, including trachoma, ophthalmia neonatorum and adult conjunctivitis.

\footnotetext{
References

${ }^{1}$ Allansmith MR: Defence of the ocular surface. Int Ophthalmol Clin 1979, 19: 93-109.

${ }^{2}$ Fleming A: On a remarkable bacteriolytic element found in tissues and secretions. Proc Roy Soc 1922, 43: 306-17.

${ }^{3}$ Thompson, $\mathrm{R}$ and Gallardo E: The antibacterial action of tears on staphylococci. Am JOphthalmol 1941, 34: 635-40.

${ }^{4}$ Friedland BR, Anderson DR, Foster RK: Non-lysozyme antibacterial factor in human tears. $A m \mathrm{~J}$ Ophthalmol 1972, 74: 52-9.
}

${ }^{5}$ Ford LC, Delange RJ, Petty RW: Identification of a nonlysozymal bacterial factor ( $\beta$-lysin) in human tears and aqueous humour. Am J Ophthalmol 1976, 81: 30-3.

${ }^{6}$ Jensen RS, Tew SG, Donaldson DM: Extracellular beta-lysin and muramidase in body fluids and inflammatory exudates. Proc Soc Broc Med 1967, 124: 545-9.

${ }^{7}$ Broekhuyse RM: Tear lactoferrin-a bacteriostatic and complexing protein. Invest Opthalmol 1974, 13: $550-4$.

${ }^{8}$ Selsted ME and Rafael J: Isolation and purification of bactericides from human tears. Exp Eye Res 1982, 34: 305-18.

${ }^{9}$ Wølner-Hansen P, Weström L, Mårdh P-A: Influence of amniotic fluid on the formation of chlamydial inclusions in McCoy cell cultures. In Mårdh P-A et al. eds. Amsterdam: Elsevier Biomed Press. 1982, 283-286.

${ }^{10}$ Elbagir AN, Ching C, Osman N, Axemo P, Bergström S, Mårdh P-A: Notes on antichlamydial activity of amniotic fluid. In Mårdh, P-A et al., eds. Proceedings of the European Society for Chlamydial Research.Stockholm: Almqvist \& Wiksell International. 1988, 128.

${ }^{11}$ Mårdh P-A, Colleen S, Sylwan J: Inhibitory effect on the formation of chlamydial inclusion in McCoy cells by seminal fluid and some of its components. Invest Urol 1980, 17: 510-13.

${ }^{12}$ Ripa KT and Mårdh P-A: Cultivation of Chlamydia trachomatis in cycloheximide-treated McCoy cells. J Clin Microbiol 1977, 6: 328-31.

${ }^{13}$ Treharne JD, Darougar S, Jones BR: Modification of microimmunofluorescence test to provide a routine serodiagnostic test for chlamydial infections. $J$ Clin Pathol 1977, 30: 510-17.

${ }^{14}$ Robert NS, James JF, Farris RL, Irwin DM: The effect of collection technique on tear composition. Invest Olphthal Vis SCi 1984, 25: 374-7.

${ }^{15}$ Soltesz V, Zeeberg B, Mårdh P-A: Toxic effect of sampling materials on the reproduction of Chlamydia trachomatis in McCoy cell cultures. Proceedings of the SSGM 5th meeting. 1988, Abs 16.

${ }^{16}$ Hackstadt T: Identification and properties of chlamydial peptides that bind eucaryotic cell surface components. J Bacteriol 1986, 13-20.

${ }^{17}$ Kueppers F: Proteinase inhibitors in human tears. Biochem Biophys Acta 1971, 229: 845-9.

${ }^{18}$ Carrol SF and Martinez RJ: Role of rabbit lysozyme in vitro serum and plasma serum bactericidal reactions against Bacillius subtilis. Infect Immun 1979, 25: 810-19.

${ }^{19}$ Sugarman B and Agbor P: The binding of Chlamydia trachomatis and zinc to McCoy cells (mouse fibroblasts). Infection 1987, 15: 39-43. 